В Е С Т Н К ПНИ П У

DOI: $10.15593 / 2224-9877 / 2016.1 .04$

УДК 621.746.628.4

\author{
И.В. Чуманов ${ }^{1}$, К.В. Шаров ${ }^{2}$, А.В. Богомягков ${ }^{2}$, \\ В.И. Васенин ${ }^{2}$, Д.О. Пустовалов ${ }^{2}$, Н.В. Мазунина ${ }^{2}$ \\ ${ }^{1} Ю$ жно-Уральский государственный университет \\ (национальный исследовательский университет), Челябинск, Россия \\ ${ }^{2}$ Пермский национальный исследовательский \\ политехнический университет, Пермь, Россия
МЕТОДИКА РАСЧЕТА НЕЗАПОЛНЕННОЙ ЯРУСНОЙ ЛИТНИКОВОЙ СИСТЕМЫ

\begin{abstract}
Ярусная литниковая система с прямым стояком широко применяется в литейном производстве. Для того чтобы данная система выполняла свои функции, необходимо обеспечить последовательное включение питателей в работу. Проектирование литниковой системы затруднено в связи с тем, что приходится прибегать к методу последовательных приближений, а также в связи с отсутствием данных о коэффициентах гидравлических сопротивлений. В статье приведена методика расчета незаполненной ярусной литниковой системы. Сначала рассчитывается оптимальное время заполнения формы. Затем определяются размеры узкого места. На начальных этапах проектирования принимается табличная величина коэффициента расхода и определяются предварительные размеры тех элементов литниковой системы, которые нельзя определить конструктивно. Затем при помощи уравнения Бернулли определяется точное значение коэффициента расхода и размеры литниковой системы пересчитываются. Так продолжается до тех пор, пока принятое значение не совпадет с достаточной точностью с полученным. Обобщены данные о величинах коэффициентов гидравлических сопротивлений различных элементов литниковых систем, требующиеся при расчете. Данная методика применена для отливки «Изложница», проведено моделирование заливки в программном комплексе ProCAST. Результаты моделирования подтвердили правильность методики. Удалось обеспечить последовательное включение питателей в работу: сначала форма заполняется через нижний питатель до тех пор, пока он не затопится и металл в форме не достигнет определенного уровня. Затем металл начинает поступать и через верхний питатель, снабжая верхнюю часть отливки горячим металлом, обеспечивая тем самым направленную кристаллизацию.
\end{abstract}

Ключевые слова: расчет ярусной литниковой системы, коэффициент расхода, стояк, питатель, чаша, коэффициент гидравлического сопротивления, скорость потока, расход, уравнение Бернулли, потери напора. 


\author{
I.V. Chumanov ${ }^{1}$, K.V. Sharov ${ }^{2}$, A.V. Bogomiagkov ${ }^{2}$, \\ V.I. Vasenin ${ }^{2}$, D.O. Pustovalov ${ }^{2}$, N.V. Mazunina ${ }^{2}$ \\ ${ }^{1}$ South Ural State University (National Research University), \\ Cheliabinsk, Russian Federation \\ ${ }^{2}$ Perm National Research Polytechnic University, Perm, Russian Federation
}

\title{
METHOD OF CALCULATION UNFILLED STEP GATING SYSTEM
}

Step gating system with direct sprue is widely used in the foundry industry. In order for this system to fulfill its function, it is necessary to ensure the consistent inclusion of feeders in work. Designing gating system is complicated due to the fact that we have to resort to the method of successive approximations, and in the absence of data on the coefficients of hydraulic resistance. The article describes the method of calculating the unfilled step gating system. First, calculate the optimal time filling of the form. Next, determine the dimensions of the narrow section. First, a tabular value of the coefficient of flow and pre-determined dimensions of those elements of gating system, which cannot be defined constructively. Then, using the Bernoulli equation to determine the exact value of the discharge coefficient, and the size of the gating system are translated. This continues until the received value matches the sufficient precision obtained. The article summarizes the data values of the coefficients of hydraulic resistance gating systems of the various elements required for the calculation. This technique is used for casting "Mould" simulated filling in the software package "ProCAST". The simulation results confirmed the correctness of methodology. It was possible to provide a series connection of feeders in operation - first form is filled through the lower feeder so long until it is flooded, and the metal in the form reaches a certain level. Then, the metal begins to flow through the upper and feeder, provided with an upper portion of the hot metal castings, thereby providing directional solidification.

Keywords: step gating system design, discharge coefficient, sprue, feeder, pouring basin, hydraulic resistance coefficient, liquid flow rate, discharge, Bernoulli equation, head looses.

\section{Введение}

Основная задача литниковой системы - обеспечить получение качественных отливок.

При выборе мест подвода металла следует руководствоваться следующими принципами:

- конструктивными особенностями отливки;

- свойствами расплава;

- принципом равномерной или направленной кристаллизации.

Для протяженных по высоте отливок оптимальным является подвод металла на нескольких уровнях.

Ярусная литниковая система обладает следующими преимуществами:

- более спокойное поступление металла в форму;

- ниже опасность размытия стенок формы; 
- в верхнюю часть отливки и в прибыли поступает более горячий металл.

Для отливок средней высоты подвод металла осуществляется на двух ярусах [1].

Есть три варианта заполнения формы через литниковую систему с прямым стояком (рис. 1).

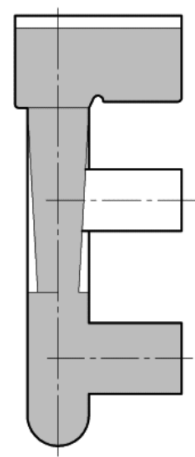

a

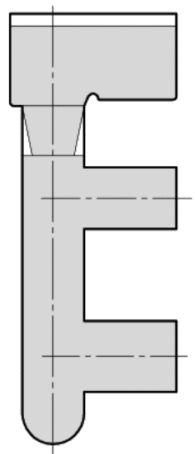

б

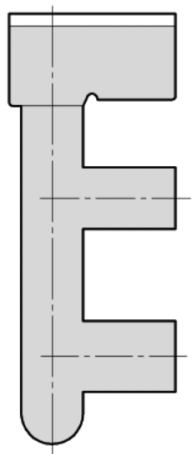

B

Рис. 1. Варианты заполнения стояка

В первом случае (рис. $1, a$ ) на начальном этапе заполнен только нижний питатель, верхний питатель вступает в действие после поднятия уровня металла в форме выше первого питателя. Во втором и третьем случаях (рис. 1, б и в) питатели вступают в работу практически одновременно, что препятствует получению годной отливки. Следовательно, литниковую систему требуется спроектировать таким образом, чтоб обеспечивалось последовательное включение питателей в работу.

\section{Методика расчета}

Расчет ярусной литниковой системы начинается с определения оптимального времени заливки $\tau$. Оно вычисляется по выражению [1]:

$$
\tau=\left(\frac{\pi W_{0} \rho C_{\mathrm{p}}\left(t_{3}-t_{\text {л }}\right)}{S_{0} b \Theta_{3}}\right)^{2},
$$

где $W_{0}$ - объем отливки, ${ }^{3} ; \rho-$ плотность материала отливки в жидком состоянии, кг $/ \mathrm{m}^{3} ; C_{\mathrm{p}}$ - удельная теплоемкость расплава (в жидком состоянии $),$ Дж/(кг. $\left.{ }^{\circ} \mathrm{C}\right) ; t_{3}-$ температура заливки расплава, ${ }^{\circ} \mathrm{C} ; t_{\text {л }}-$ температура ликвидус, ${ }^{\circ} \mathrm{C} ; S_{0}-$ площадь контакта отливки с формой, ${ }^{2}$; 
$b$ - коэффициент аккумуляции теплоты для материала формы, Дж/(м'c $\left.\cdot{ }^{\circ} \mathrm{C}\right) ; \Theta_{3}-$ относительная температура заливки, $\Theta_{3}=\left(t_{\phi}-t_{3}\right), t_{\phi}-$ начальная температура формы.

Также рекомендуется формула [2]

$$
\tau=A_{i} M^{0,4}
$$

где $A_{i}-$ эмпирический коэффициент, зависящий от сплава; $M$ - масса отливки, кг.

Величины коэффициентов $A_{i}[3]$ :

\begin{tabular}{|c|c|c|c|c|c|}
\hline Сплав & СЧ, ВЧ, БЧ & $\begin{array}{c}\text { Углеродистая } \\
\text { сталь }\end{array}$ & $\begin{array}{c}\text { Сплавы на основе } \\
\text { алюминия }\end{array}$ & $\begin{array}{c}\text { Сплавы на } \\
\text { основе магния }\end{array}$ & $\begin{array}{c}\text { Сплавы на } \\
\text { основе меди }\end{array}$ \\
\hline$A_{i}$ & 3,7 & 2,4 & 4,5 & 5,0 & 3,5 \\
\hline
\end{tabular}

Для черных металлов Дубицкий предлагает использовать формулу, выведенную из номограммы Соболева [4]:

$$
\tau=S_{1} \sqrt[3]{\delta M},
$$

где $S_{1}$ - коэффициент, зависящий от применяемого сплава; $\delta$ - толщина стенок.

После определения времени заливки $\tau$ требуется рассчитать суммарную площадь узкого сечения $\sum S_{\text {узк }}$ [4], для нашего случая узким сечением является верхнее сечение стояка $S_{2}$ :

$$
\sum S_{\text {узк }}=\frac{M}{\mu \tau \rho \sqrt{2 \mathrm{~g} H_{\mathrm{p}}}}
$$

где $\mu$ - коэффициент расхода ЛС; $\tau$ - расчетное оптимальное время заливки, с; $\rho$ - плотность жидкого металла, кг $/ \mathrm{M}^{3} ; g-$ ускорение силы тяжести, м/ $\mathrm{c}^{2} ; H_{\mathrm{p}}$ - расчетный напор, м.

Определение коэффициента $\mu$ вызывает наибольшие трудности. Во-первых, чтобы найти значение $\mu$, необходимо знать все размеры литниковой системы, а нам их и надо найти. Именно поэтому прибегают к методу последовательных приближений: сначала задаются табличным значением, получают первичные размеры литниковой системы, затем рассчитывают значение коэффициента расхода для полученной системы. Полученное значение $\mu$ подставляется в выражение (4), и расчет повторяется до тех пор, пока литниковая система не обеспечит должный расход. 
Во-вторых, для расчета необходимы значения коэффициентов местных гидравлических сопротивлений элементов литниковой системы $\zeta$, но в литературе зачастую отсутствует информация о величине некоторых коэффициентов, либо она противоречива [5-10].

В-третьих, требуется применить уравнение Бернулли для системы с раздачей потока и переменным расходом, что не доказано теоретически.

Первая стадия заполнения - работает только нижний питатель

Узким сечением при данных условиях заполнения будет являться верхнее сечение стояка 2-2 (рис. 2).

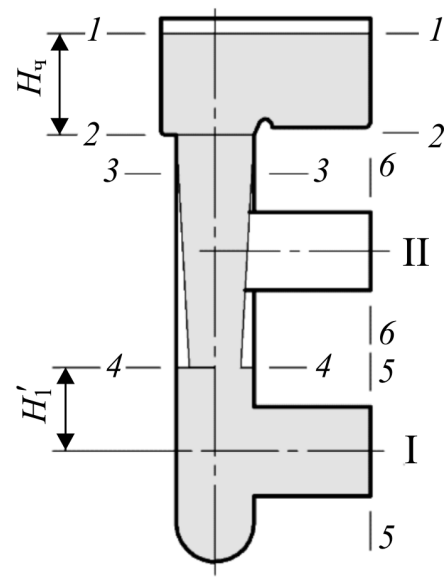

Рис. 2. Схема к расчету истечения из нижнего питателя

Расчетный напор будет равен высоте уровня металла в чаше $H_{4}$. Сечение нижнего питателя определяется по формуле

$$
S_{5}=S_{2} \frac{\mu_{\mathrm{u}} \sqrt{H_{\mathrm{u}}}}{\mu_{4-5} \sqrt{H_{1}^{\prime}}},
$$

где $S_{5}$ и $S_{2}$ - площади соответствующих поперечных сечений, м²; $\mu_{4}-$ коэффициент расхода чаши; $\mu_{4-5}-$ коэффициент расхода в нижнем питателе; $H_{1}^{\prime}$ - напор, действующий в стояке, м. Высотой $H_{1}^{\prime}$ необходимо задаться, но она должна быть меньше расстояния между верхним и нижним питателем. Коэффициент расхода чаши для цветных сплавов может быть найден по таблице либо определен по выражению [6] 


$$
\mu_{\mathrm{ч}}=\frac{1}{\sqrt{1+\zeta_{\mathrm{ч}}}}
$$

где $\zeta_{\text {ч }}-$ коэффициент местного сопротивления на вход из чаши в стояк.

Значения коэффициентов расхода чаши для цветных сплавов [11]

\begin{tabular}{|c|c|c|c|}
\hline $\begin{array}{l}\text { Диаметр выходного } \\
\text { отверстия в чаше, мм }\end{array}$ & $H_{\mathrm{u}}, \mathrm{MM}$ & $t_{3},{ }^{\circ} \mathrm{C}$ & $\mu_{\mathrm{u}}$ \\
\hline \multirow[t]{6}{*}{ 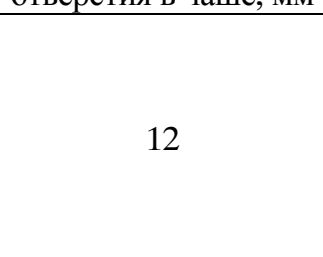 } & 70 & \multirow{3}{*}{720} & 0,980 \\
\hline & 150 & & 0,970 \\
\hline & $200-280$ & & 0,965 \\
\hline & \multirow{5}{*}{150} & 680 & 0,950 \\
\hline & & 760 & 0,975 \\
\hline & & 800 & 0,980 \\
\hline 20 & & \multirow{2}{*}{720} & 0,975 \\
\hline 30 & & & 0,985 \\
\hline
\end{tabular}

Значения коэффициента местного сопротивления на вход из чаши в стояк [7]:

\begin{tabular}{|c|c|c|c|c|c|c|c|}
\hline$r / d_{\text {cr }}{ }^{*}$ & 0,00 & 0,01 & 0,02 & 0,05 & 0,10 & 0,16 & $\geq 0,20$ \\
\hline$\zeta_{\text {cr }}$ & 0,50 & 0,43 & 0,36 & 0,22 & 0,10 & 0,06 & 0,03 \\
\hline
\end{tabular}

" $r$-радиус скругления на входе из чаши в стояк, мм; $d_{\text {ст }}-$ диаметр стояка, мм.

Коэффициент расхода $\mu_{4-5}$ определяется методом последовательных приближений.

Для этого составим уравнение Бернулли для сечений 4-4 и 5-5:

$$
\frac{p_{4}}{\gamma}+\alpha \frac{v_{4}^{2}}{2 g}+H_{1}^{\prime}=\frac{p_{5}}{\gamma}+\alpha \frac{v_{5}^{2}}{2 g}+h_{4-5}^{(1)},
$$

где $p_{4}$ и $p_{5}-$ давление в соответствующих сечениях, Па; $v_{4}$ и $v_{5}-$ скорости потока в соответствующих питателях, м/с; $\gamma$ - удельный вес жидкого металла, кг $/ \mathrm{M}^{3} ; \alpha-$ коэффициент неравномерности распределения скорости по сечению потока (коэффициент Кориолиса); $g$ - ускорение свободного падения, $\mathrm{m} / \mathrm{c}^{2}$.

Давления $p_{4}$ и $p_{5}$ равны между собой, так как сечение 4-4 связано с полостью формы через незаполненный питатель. Принимая это во внимание, а также расписывая потери напора, из выражения (7) получим

$$
\alpha \frac{v_{4}^{2}}{2 g}+H_{1}^{\prime}=\alpha \frac{v_{5}^{2}}{2 g}+\lambda \frac{H_{1}^{\prime}}{d_{\text {ст }}} \cdot \alpha \frac{v_{4}^{2}}{2 g}+\zeta_{\text {пов }} \cdot \alpha \frac{v_{5}^{2}}{2 g}+\lambda \frac{l_{\text {пI }}}{d_{\text {пI }}} \cdot \alpha \frac{v_{5}^{2}}{2 g},
$$


где $\lambda$ - коэффициент потерь на трение; $d_{\text {ст }}-$ диаметр стояка, м; $\zeta_{п о в}-$ коэффициент местного сопротивления на поворот из стояка в питатель; $l_{\text {пI }}$ - длина питателя I, м; $d_{\text {пI }}$ - диаметр питателя I, м. Отдельно следует заметить, что для литниковых каналов некруглых поперечных сечений при расчетах используют гидравлический диаметр, который находится по выражению [6]

$$
d_{\Gamma}=\frac{4 S}{P}
$$

где $S$ - площадь поперечного сечения элемента, ${ }^{2} ; P$ - смоченный периметр, м.

Исследования величины коэффициента местного сопротивления на поворот проводились в работе [5], результаты представлены ниже.

Значения коэффициента местного сопротивления на поворот на $90^{\circ}[5]:$

\begin{tabular}{|c|c|}
\hline Диапазон $S_{\text {п }} / S_{\text {к }}$ или $S_{\text {K }} / S_{\text {ст }}$ & $\zeta_{\text {пов }}$ \\
\hline $0,025-0,251$ & $0,280+0,115 S_{\Pi} / S_{\mathrm{K}}$ \\
\hline $0,251-1$ & $0,557\left(S_{\mathrm{\Pi}} / S_{\mathrm{K}}\right)^{2}+0,066 S_{\mathrm{\Pi}} / S_{\mathrm{K}}+0,275$ \\
\hline 1 & 0,885 \\
\hline $1-3,985$ & $1,273\left(S_{\mathrm{K}} / S_{\mathrm{cr}}\right)^{2}-0,746 S_{\mathrm{K}} / S_{\mathrm{cт}}+0,353$ \\
\hline $3,985-15,821$ & $1,053\left(S_{\mathrm{K}} / S_{\mathrm{cT}}\right)^{2}-0,658 S_{\mathrm{K}} / S_{\text {ст }}+3,502$ \\
\hline
\end{tabular}

Выразим скорость $v_{4}$ через $v_{5}$ из уравнения неразрывности, которое для данного случая будет иметь вид

$$
v_{4} S_{4}=v_{5} S_{5}
$$

Получаем

$$
v_{4}=v_{5} \frac{S_{5}}{S_{4}}
$$

Тогда выражение (8) можно записать как

$$
H_{1}^{\prime}=\alpha \frac{v_{5}^{2}}{2 g}\left[\left(\lambda \frac{H_{1}^{\prime}}{d_{\text {ст }}}-1\right)\left(\frac{S_{5}}{S_{4}}\right)^{2}+\zeta_{\text {пов }}+\lambda \frac{l_{\text {пI }}}{d_{\text {пI }}}+1\right] .
$$

Обозначим часть выражения (11) в квадратных скобках как

$$
\zeta_{4-5(5)}^{(1)}=\left(\lambda \frac{H_{1}^{\prime}}{d_{\text {ст }}}-1\right)\left(\frac{S_{5}}{S_{4}}\right)^{2}+\zeta_{\text {пов }}+\lambda \frac{l_{\text {пI }}}{d_{\text {пI }}}+1 .
$$


Коэффициент расхода литниковой системы при работе только нижнего питателя будет [6]

$$
\mu_{4-5(5)}^{(1)}=\frac{1}{\sqrt{1+\zeta_{4-5(5)}^{(1)}}} .
$$

Затем подставляем полученные значения в формулу (4) и повторяем расчет до тех пор, пока результат не будет отличаться от принятого значения на приемлемую величину.

\section{Работают оба питателя}

На следующей стадии заполнения стояк полностью заполнен расплавом (рис. 3) и работают оба питателя. Расчет ведем по аналогии с работами $[12,13]$.

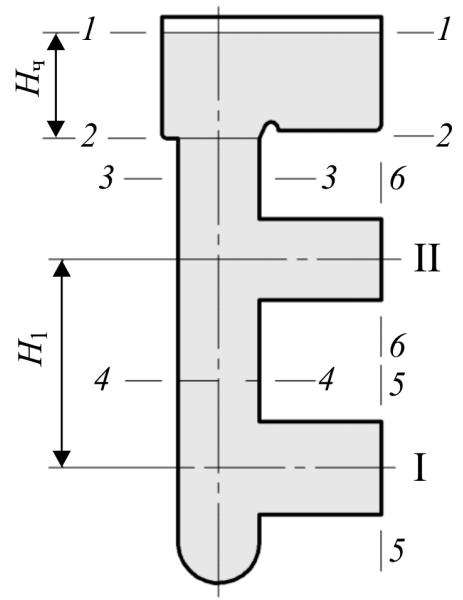

Рис. 3. Схема к расчету истечения из обоих питателей

Для расчета составим уравнения Бернулли для сечений 1-1 и 5-5:

$$
\begin{aligned}
H+\frac{p_{1}}{\gamma}+\alpha \frac{v_{1}^{2}}{2 g} & =\left(\zeta_{\text {сг }}+\lambda \frac{l_{\text {ст }}}{d_{2}}\right) \alpha \frac{v_{2}^{2}}{2 g}+\left(\zeta_{3-4(4)}^{\text {M }}+\lambda \frac{H_{1}}{d_{4}}\right) \cdot \alpha \frac{v_{4}^{2}}{2 g}+ \\
& +\left(\zeta_{\text {п1 }}+\lambda \frac{l_{\mathrm{\Pi I}}}{d_{\mathrm{\Pi I}}}+1\right) \alpha \frac{v_{5}^{2}}{2 g}+\frac{p_{5}}{\gamma},
\end{aligned}
$$

и для сечений 1-1 и 6-6: 


$$
\begin{gathered}
H-H_{1}+\frac{p_{1}}{\gamma}+\alpha \frac{v_{1}^{2}}{2 g}=\left(\zeta_{\text {ст }}+\lambda \frac{l_{\text {ст }}-H_{1}}{d_{2}}\right) \alpha \frac{v_{2}^{2}}{2 g}+ \\
+\left(\zeta_{3-6(6)}+\lambda \frac{l_{\text {пII }}}{d_{\text {пाI }}}+1\right) \cdot \alpha \frac{v_{6}^{2}}{2 g}+\frac{p_{6}}{\gamma},
\end{gathered}
$$

где $\zeta^{\text {д }} 3-4$ (4) - коэффициент сопротивления на проход потока [14],

$$
\zeta_{3-4(4)}^{\text {д }}=0,4\left(1-v_{3} / v_{4}\right)^{2} /\left(v_{3} / v_{4}\right)^{2},
$$

a $\zeta_{3-6(6)}^{\text {д }}$ коэффициент сопротивления на ответвление части потока в питатель [14],

$$
\zeta_{3-6(6)}^{\text {д }}=\left[1+\varphi\left(v_{6} / v_{3}\right)^{2}\right] /\left(v_{6} / v_{3}\right)^{2}
$$

где $\varphi$ - коэффициент, зависящий от соотношения площадей.

$$
\begin{array}{r}
H=\left(\zeta_{\text {ст }}+\lambda \frac{l_{\text {ст }}}{d_{2}}\right) \alpha \frac{v_{2}^{2}}{2 g}+\left(\zeta_{3-4(4)}^{\text {д }}+\lambda \frac{H_{1}}{d_{4}}\right) \cdot \alpha \frac{v_{4}^{2}}{2 g}+\left(\zeta_{\text {пов }}+\lambda \frac{l_{\text {пI }}}{d_{\text {пI }}}+1\right) \alpha \frac{v_{5}^{2}}{2 g}, \\
H-H_{1}=\left(\zeta_{\text {ст }}+\lambda \frac{l_{\text {ст }}-H_{1}}{d_{2}}\right) \alpha \frac{v_{2}^{2}}{2 g}+\left(\zeta_{3-6(6)}^{\text {д }}+\lambda \frac{l_{\text {пII }}}{d_{\text {пII }}}+1\right) \cdot \alpha \frac{v_{6}^{2}}{2 g} .
\end{array}
$$

Обозначим отношения скоростей в питателях как $x_{2}$, а отношение площадей - как $y_{2}$. Тогда уравнение неразрывности для двух работающих питателей примет вид

$$
\begin{gathered}
v_{3} S_{3}=v_{4} S_{4}+v_{6} S_{6}=v_{5} S_{5}+v_{6} S_{6}=v_{5} S_{5}+\frac{v_{5}}{x_{2}} \cdot \frac{S_{5}}{y_{2}}= \\
=v_{5} S_{5}\left(1+\frac{1}{x_{2} y_{2}}\right)=v_{5} S_{\text {пр }(5)}^{(2)},
\end{gathered}
$$

где $S_{\text {пр(5) }}^{(2)}$ - приведенная к скорости $v_{5}$ площадь питателей (для двух работающих питателей);

$$
v_{5} S_{5}+v_{6} S_{6}=x_{2} v_{6} y_{2} S_{6}+v_{6} S_{6}=v_{6} S_{6}\left(x_{2} y_{2}+1\right)=v_{6} S_{\text {пр }(6)}^{(2)},
$$

где $S_{\text {пр(6) }}^{(2)}$ - приведенная к скорости $v_{6}$ площадь питателей (для двух работающих питателей). 
Требуется найти отношение скоростей $v_{3} / v_{4} ; v_{6} / v_{3}$. Находим их из выражений (20) и (21):

$$
\begin{gathered}
v_{3} / v_{4}=\left(1+\frac{1}{x_{2} y_{2}}\right)\left(\frac{S_{4}}{S_{3}}\right), \\
v_{6} / v_{3}=\frac{S_{3}}{S_{6}\left(x_{2} y_{2}+1\right)} .
\end{gathered}
$$

Величиной $x_{2}$ задаемся произвольно. Тогда (18) и (19) можно записать как

$$
\begin{aligned}
& H=\alpha \frac{v_{5}^{2}}{2 g}\left[\left(\zeta_{\text {ст }}+\lambda \frac{l_{\text {ст }}}{d_{2}}\right)\left(\frac{S_{\text {пр }(5)}^{(2)}}{S_{2}}\right)^{2}+\left(\zeta_{3-4(4)}^{\text {म }}+\lambda \frac{H_{1}}{d_{4}}\right)\left(\frac{S_{5}}{S_{4}}\right)^{2}+\zeta_{\text {пов }}+\lambda \frac{l_{\text {пI }}}{d_{\text {пI }}}+1\right], \\
& H-H_{1}=\alpha \frac{v_{6}^{2}}{2 g}\left[\left(\zeta_{\mathrm{cr}}+\lambda \frac{l_{\text {ст }}-H_{1}}{d_{2}}\right)\left(\frac{S_{\mathrm{rp}(6)}^{(2)}}{S_{2}}\right)^{2}+\zeta_{3-6(6)}^{\mathrm{L}}+\lambda \frac{l_{\mathrm{nIII}}}{d_{\mathrm{nII}}}+1\right] .
\end{aligned}
$$

Выражение в квадратных скобках в формуле (22) обозначим как $\zeta_{1-5(5)}^{(2)}$, а в (23) - как $\zeta_{1-6(6)}^{(2)}$. Затем по выражению (13) определяем коэффициенты расхода $\mu_{1-5(5)}^{(2)}$ и $\mu_{1-6(6)}^{(2)}$. Зная эти коэффициенты, находим скорости $v_{5}$ и $v_{6}$ :

$$
v_{i}=\mu_{1-i(i)}^{(1)} \sqrt{\frac{2 g H_{\text {д }}}{\alpha}},
$$

где $H_{д}-$ напор, действующий на данном уровне. Для нижнего питателя $H_{\text {д }}=H$, для верхнего $H_{\text {д }}=H-H_{1}$.

После того как скорости найдены, вычисляем их отношение и сравниваем его с $x_{2}$. В случае несовпадения повторяем расчет, принимая за $x_{2}$ полученное отношение. И так повторяем расчет до тех пор, пока принятое и полученное значения не будут отличаться на приемлемую величину.

\section{Проверка методики}

Для проверки разработанной методики сконструируем литниковую систему для чугунной отливки «Изложница» массой $M=5300$ кг 
и смоделируем процесс заполнения формы металлом в программном комплексе ProCAST. Схема подвода металла представлена на рис. 4.

Оптимальное время заливки $\tau=3,7 \cdot 5300^{0,4}=114,27 \mathrm{c}$.

Площадь $S_{2}$ и диаметр $d_{\text {ст: }}: S_{2}=0,00388124 \mathrm{~m}^{2}=38,8124 \mathrm{~cm}^{2}, d_{\mathrm{cт}}=$ $=0,0703 \mathrm{м}=70,3 \mathrm{MM} ; S_{5}=0,0054808 \mathrm{~m}^{2}=54,808 \mathrm{~cm}^{2} ; d_{\Pi \mathrm{I}}=0,0835 \mathrm{M}=$ $=83,5 \mathrm{MM} ; \mu_{4-5(5)}^{(1)}=0,532$.

Погрешность определения коэффициента расхода $E_{\mu}=0,0008575$.

При работе двух питателей принимаем, что $S_{6}=0,8 S_{5}$, и при заданном $x_{2}=1,8393$ находим $\mu_{1-5(5)}^{(2)}=0,211892 ; \mu_{1-6(6)}^{(2)}=0,145395 ; v_{5}=$ $=1,047437 \mathrm{M} / \mathrm{c} ; v_{6}=0,569446 \mathrm{M} / \mathrm{c}$.

Подробнее о методе последовательных приближений написано в работах $[12,13]$.

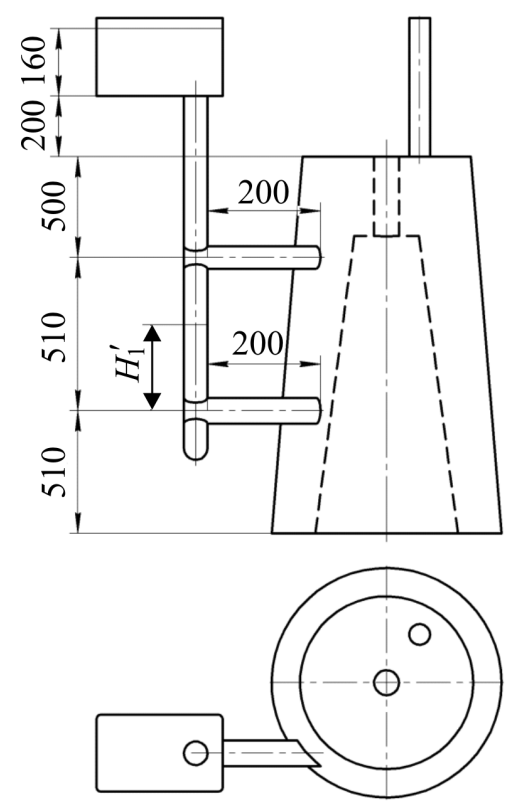

Рис. 4. Схема подвода металла к отливке

Для моделирования была построена 3D-модель с дополнительным резервуаром для слива избытков жидкости для поддержания постоянной величины напора [15]. Ниже показаны результаты моделирования, на которых виден уровень $H_{1}^{\prime}$ (рис. $5, a$ ), которым мы задавались при расчетах, а также уровень металла в форме при включении в работу верхнего питателя (рис. 5, б). 


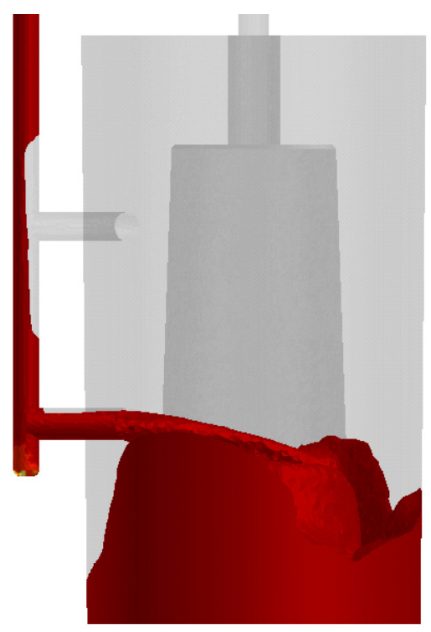

$a$

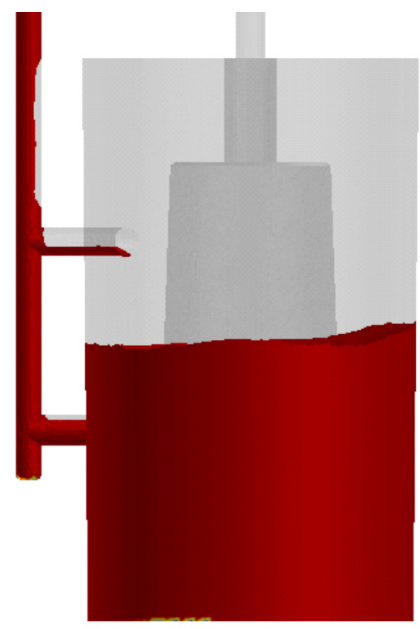

6

Рис. 5. Моделирование первой $(a)$ и второй (б) стадий заполнения

\section{Заключение}

Спроектированная литниковая система работает должным образом. Поддерживается заданный уровень металла в стояке. Верхний питатель включается в работу после затопления нижнего. Замечен незначительный захват металла верхним питателем. Это устраняется наклонным расположением верхнего питателя. Также при моделировании видны моменты, когда питатель заполнен не до конца. Это снижает точность расчетов, но она выше, чем при простой подстановке табличного значения без дальнейших итераций.

\section{Список литературы}

1. Литье по выплавляемым моделям / В.Н. Иванов, С.А. Казеннов, Б.С. Курчман [и др.]; под ред. Я.И. Шклейника, В.А. Озерова. - 3-е изд., перераб. и доп. - М.: Машиностроение, 1984. - 408 с.

2. Рабинович Б.В. Введение в литейную гидравлику. - М.: Машиностроение, 1966. - 443 с.

3. Технология литейного производства / А.П. Трухов, Ю.А. Сорокин, М.Ю. Ершов [и др.]; под ред. А.П. Трухова. - М.: Академия, 2005. $-528 \mathrm{c}$.

4. Дубицкий Г.М. Литниковые системы. - М.: Свердловск: Машгиз, 1962. $-256 \mathrm{c}$. 
5. Исследование местных сопротивлений литниковой системы / В.И. Васенин, Д.В. Васенин, А.В. Богомягков, К.В. Шаров // Вестник Пермского национального исследовательского политехнического университета. Машиностроение, материаловедение. - 2012. - Т. 14, № 2. C. $46-53$.

6. Чугаев Р.Р. Гидравлика. - М.: Бастет, 2008. - 672 с.

7. Идельчик И.Е. Справочник по гидравлическим сопротивлениям. - М.: Машиностроение, 1992. - 672 с.

8. Вильнер Я.М., Ковалёв Я.Т., Некрасов Б.Б. Справочное пособие по гидравлике, гидромашинам и гидроприводам. - Минск: Вышэйш. шк., 1976. - 416 с.

9. Справочник по гидравлическим расчетам / П.Г. Киселев, А.Д. Альтшуль, Н.В. Данильченко [и др.]; под ред. П.Г. Киселева. М.: Энергия, 1972. - 312 с.

10. Альтшуль А.Д., Животовский Л.С., Иванов Л.П. Гидравлика и аэродинамика. - М.: Стройиздат, 1987. - 416 с.

11. Цветное литье: справочник / Н.М. Галдин, Д.Ф. Чернега, Д.Ф. Иванчук [и др.]; под ред. Н.М. Галдина. - М.: Машиностроение, 1989. $-528 \mathrm{c}$.

12. Шаров К.В. Исследование ярусной литниковой системы с питателями различных площадей поперечных сечений [Электронный ресурс] // Современные проблемы науки и образования. - 2014. № 6. - URL: http://www.science-education.ru/120 (дата обращения: 20.11.2015).

13. Васенин В.И. Исследование L-образной литниковой системы // Литейное производство. - 2011. - № 8. - С. 32-35.

14. Меерович И.Г., Мучник Г.Ф. Гидродинамика коллекторных систем. - М.: Наука, 1986. - 144 с.

15. Методика моделирования течения расплава в литниковой системе с постоянным напором / Д.О. Пустовалов, Ю.А. Набокова, К.А. Мазуренко, К.В. Шаров, А.А. Шумков // Известия Самар. науч. центра РАН. 2015. - T. 17, № 2(4). - C. 926-928.

\section{References}

1. Ivanov V.N., Kazennov S.A., Kurchman B.S. Lit'e po vyplavliaemym modeliam [Lost Wax Casting]. Moscow: Mashinostroenie, 1984. 408 p. 
2. Rabinovich B.V. Vvedenie v liteinuiu gidravliku [Introduction into the casting hydraulics]. Moscow: Mashinostroenie, 1966. 443 p.

3. Trukhov A.P., Sorokin Iu.A., Ershov M.Iu. Tekhnologiia liteinogo proizvodstva [Foundry technology]. Moscow: Akademiia, 2005. 528 p.

4. Dubitskii G.M. Litnikovye sistemy [Gating system]. Moscow: Sverdlovsk: Mashgiz, 1962. 256 p.

5. Vasenin V.I., Vasenin D.V., Bogomiagkov A.V., Sharov K.V. Issledovanie mestnykh soprotivlenii litnikovoi sistemy [A study of local resistance gating system] Vestnik Permskogo natsional'nogo issledovatel'skogo politekhnicheskogo universiteta. Mashinostroenie, materialovedenie, 2012, vol. 14, no. 2, pp. 46-53.

6. Chugaev R.R. Gidravlika [Hydraulics]. Moscow: Bastet, 2008. 672 p.

7. Idel'chik I.E. Spravochnik po gidravlicheskim soprotivleniiam [Manual hydraulic resistance]. Moscow: Mashinostroenie, 1992. $672 \mathrm{p}$.

8. Vil'ner Ia.M., Kovalev Ia.T., Nekrasov B.B. Spravochnoe posobie po gidravlike, gidromashinam i gidroprivodam [Handbook on hydraulics, hydraulic machines and hydraulicdrives]. Minsk: Vysheishaia shkola, 1976. $416 \mathrm{p}$.

9. Kiselev P.G., Al'tshul' A.D., Danil'chenko N.V. Spravochnik po gidravlicheskim raschetam [Manual hydraulic calculation]. Moscow: Energiia, $1972.312 \mathrm{p}$.

10. Al'tshul' A.D., Zhivotovskii L.S., Ivanov L.P. Gidravlika i aerodinamika [Hydraulics and aerodynamics]. Moscow: Stroiizdat, 1987. 416 p.

11. Galdin N.M., Chernega D.F., Ivanchuk D.F. Tsvetnoe lit'e: spravochnik [Color Cast: A Handbook]. Moscow: Mashinostroenie, 1989. 528 p.

12. Sharov K.V. Issledovanie iarusnoi litnikovoi sistemy s pitateliami razlichnykh ploshchadei poperechnykh sechenii [Research bunk feeders gating system with different cross-sectional areas]. Sovremennye problemy nauki i obrazovaniia, 2014, no. 6, available at: http://www.science-education.ru/120 (accessed 20 November 2015).

13. Vasenin V.I. Issledovanie L-obraznoi litnikovoi sistemy [Investigation of L-shaped runner system]. Liteinoe proizvodstvo, 2011, no. 8, pp. 32-35.

14. Meerovich I.G., Muchnik G.F. Gidrodinamika kollektornykh system [Hydrodynamics collection systems]. Moscow: Nauka, 1986. 144 p.

15. Pustovalov D.O., Nabokova Iu.A., Mazurenko K.A., Sharov K.V., Shumkov A.A. Metodika modelirovaniia techeniia rasplava $v$ litnikovoi sis- 
teme s postoiannym naporom [Methods of melt flow simulation in the gating system with constant pressure]. Izvestiia Samarskogo nauchnogo tsentra Rossiiskoi akademii nauk, 2015, vol. 17, no. 2(4), pp. 926-928.

Получено 29.10.2015

\section{Об авторах}

Чуманов Илья Валерьевич (Челябинск, Россия) - доктор технических наук, профессор, заведующий кафедрой «Техника и технология производства материалов» Южно-Уральского государственного университета (национального исследовательского университета); e-mail: chiv71@susu.ac.ru.

Шаров Константин Владимирович (Пермь, Россия) - старший преподаватель кафедры «Материалы, технологии и конструкции машин» Пермского национального исследовательского политехнического университета; e-mail: ksharov@yandex.ru.

Богомягков Алексей Васильевич (Пермь, Россия) - старший преподаватель кафедры «Материалы, технологии и конструкции машин» Пермского национального исследовательского политехнического университета; e-mail: bogomyagkovav@yandex.ru.

Васенин Валерий Иванович (Пермь, Россия) - кандидат технических наук, доцент кафедры «Материалы, технологии и конструкции машин» Пермского национального исследовательского политехнического университета; e-mail: detali@pstu.ru.

Пустовалов Дмитрий Олегович (Пермь, Россия) - старший преподаватель кафедры «Материалы, технологии и конструкции машин» Пермского национального исследовательского политехнического университета; e-mail: pustovalov.dmitrii@inbox.ru.

Мазунина Нина Валерьевна (Пермь, Россия) - магистрант кафедры «Материалы, технологии и конструкции машин» Пермского национального исследовательского политехнического университета; e-mail: detali@pstu.ru.

\section{About the authors}

Ilia V. Chumanov (Chelyabinsk, Russian Federation) - Doctor of Technical Sciences, Professor, Head of Department "Technique and Techno- 
logy of Materials", South Ural State University (National Research University); e-mail: chiv71@susu.ac.ru.

Konstantin V. Sharov (Perm, Russian Federation) - Senior Lecturer, Department "Materials, Technologies and Construction of Machines", Perm National Research Polytechnic University; e-mail: ksharov@yandex.ru.

Aleksei V. Bogomiagkov (Perm, Russian Federation) - Senior Lecturer, Department "Materials, Technologies and Construction of Machines", Perm National Research Polytechnic University; e-mail: bogomyagkovav@yandex.ru.

Valerii I. Vasenin (Perm, Russian Federation) - Ph. D. in Technical Sciences, Associate Professor, Department "Materials, Technologies and Construction of Machines", Perm National Research Polytechnic University; e-mail: detali@pstu.ru.

Dmitrii O. Pustovalov (Perm, Russian Federation) - Senior Lecturer, Department "Materials, Technologies and Construction of Machines", Perm National Research Polytechnic University; e-mail: pustovalov. dmitrii@inbox.ru.

Nina V. Mazunina (Perm, Russian Federation) - Master Student, Department "Materials, Technologies and Construction of Machines", Perm National Research Polytechnic University; e-mail: detali@pstu.ru. 